\title{
Arbor
}

\section{Física y Tecnologías Físicas}

\author{
José Luis Huertas Díaz
}

Arbor CLXVI, 653 (Mayo 2000), 67-82 pp.

Con este trabajo se pretende dar una visión de la situación, dentro del CSIC, de la investigación en Física y en las tecnologías afines a la Física. Se describe la estructura de institutos y centros que se dedican fundamentalmente a investigar en estas materias, introduciendo las principales lineas temáticas sobre las que se trabaja en esos institutos. En relación con esas líneas y con la demanda científica y tecnológica existente en cada una de ellas, se analiza el personal investigador y se discute su potencial desde diversas perspectivas. Finalmente se esbozan algunas reflexiones sobre el futuro del CSIC en lo relativo a este Area.

\section{Introducción}

Los estudios que el CSIC realiza en este ámbito se orientan tanto a modelar la estructura material del universo, como a aprovechar las oportunidades que se derivan del empleo de técnicas físicas y matemáticas en la resolución de problemas tecnológicos de muy diversa índole.

En este Área es posible distinguir, por tanto, entre aspectos básicos, orientados a desentrañar el entorno que nos rodea y del que formamos parte, y aspectos aplicados, encaminados a la aplicación del conocimiento a la generación y desarrollo de nuevas tecnologías de las que pueda beneficiarse el ser humano. Estas investigaciones permiten aumentar nuestro conocimiento sobre el mundo en que vivimos, además de contribuir al enunciado y resolución de importantes problemas que afectan a la calidad de vida de la sociedad. 


\section{Estructura de centros y servicios}

En los inicios de la década de los setenta, después de la reestructuración del Patronato Juan de la Cierva, se incorporan a este Area de Física y Tecnologías Físicas los primeros Institutos de cariz tecnológico. En general el conjunto de centros que formaban este ámbito del conocimiento era muy distinto de los que actualmente se encuadran en él y mostraban un panorama claramente diferente del actual. No sólo en lo referente a su organización y ubicación, sino también en cuanto a la calidad y cuantía de los equipamientos e instalaciones, al personal, a la internacionalización de las investigaciones y a su incidencia en la sociedad. Los cambios más importantes han sido, sin lugar a dudas, los derivados de la creación de institutos, ya que casi la mitad de ellos no existían hace quince años. Ocho institutos y dos laboratorios han comenzado su andadura científica en ese tiempo. Esto se traduce en que una parte muy significativa de las temáticas investigadoras del área son de gran actualidad, lo que redunda en un posicionamiento estratégico muy adecuado, como veremos más adelante.

La investigación en Física básica y en tecnologías afines se desarrolla dentro del CSIC en el conjunto de centros e institutos que se muestra en la Tabla I. En dicha Tabla se resumen algunos aspectos de especial relevancia, tales como su localización geográfica, su tamaño y su organización ${ }^{1}$. La mayor parte de estos institutos no tiene problemas importantes de espacio o equipamiento. Como puede verse, hay nueve Institutos que se integran en tres Centros, para facilitar su coordinación y cooperación. Aunque no se indica en la Tabla, los Institutos que trabajan en Astrofísica se coordinan a través de una Red, que no es sino una estructura de nivel superior que permite una relación más directa entre ellos.

TABLA 1

\begin{tabular}{||l|l|l|l|l|l||}
\hline \multicolumn{1}{|c|}{ Nombre } & \multicolumn{1}{|c|}{ Siglas } & Localización & \multicolumn{1}{c|}{ Tipo } & $\begin{array}{c}\text { Otras } \\
\text { Instituc. }\end{array}$ & Personal \\
\hline $\begin{array}{l}\text { Instituto de Estructura } \\
\text { de la Materia } \dagger\end{array}$ & IEM & Madrid & Propio & & $134(40)$ \\
\hline $\begin{array}{l}\text { Instituto de Matemáticas } \\
\text { y Física Fundamental } \dagger\end{array}$ & IMAFF & Madrid & Propio & & $52(29)$ \\
\hline \begin{tabular}{l} 
Instituto de Optica $\dagger$ \\
\hline Instituto de Acústica $\ddagger$
\end{tabular} & IO & Madrid & Propio & & $33(11)$ \\
\hline $\begin{array}{l}\text { Instituto de Física } \\
\text { Aplicada } \neq\end{array}$ & IFA & Madrid & Propio & & $62(15)$ \\
\hline
\end{tabular}




\section{Física y Tecnologías Físicas}

TABLA 1 (Continuación)

\begin{tabular}{|c|c|c|c|c|c|}
\hline Nombre & Siglas & Localización & Tipo & $\begin{array}{l}\text { Otras } \\
\text { Instituc. }\end{array}$ & Personal \\
\hline $\begin{array}{l}\text { Instituto de Automática } \\
\text { Industrial } \ddagger\end{array}$ & IAI & $\begin{array}{l}\text { Arganda } \\
\text { Madrid }\end{array}$ & Propio & & $126(20)$ \\
\hline $\begin{array}{l}\text { Instituto de Microelectrónica } \\
\text { de Barcelona * }\end{array}$ & IMB & $\begin{array}{l}\text { Bellaterra } \\
\text { (Barcelona) }\end{array}$ & Propio & $\mathrm{UAB}$ & $138(19)$ \\
\hline $\begin{array}{l}\text { Instituto de Microelectrónica } \\
\text { de Madrid * }\end{array}$ & IMM & Madrid & Propio & & $36(16)$ \\
\hline $\begin{array}{l}\text { Instituto de Microelectrónica } \\
\text { de Sevilla * }\end{array}$ & IMSE & Sevilla & Propio & USE, JA & $53(16)$ \\
\hline Instituto de Física Corpuscular & IFIC & Valencia & Mixto & UVA & $116(41)$ \\
\hline Instituto de Física de Cantabria & IFCA & Santander & Mixto & UCAN & $37(12)$ \\
\hline $\begin{array}{l}\text { Instituto de Investigación en } \\
\text { Inteligencia Artificial }\end{array}$ & IIIA & $\begin{array}{l}\text { Bellaterra } \\
\text { (Barcelona) }\end{array}$ & Propio & & $34(12)$ \\
\hline $\begin{array}{l}\text { Instituto de Robótica e Informática } \\
\text { Industrial }\end{array}$ & IRII & Barcelona & Mixto & UPC & $44(11)$ \\
\hline $\begin{array}{l}\text { Instituto de Astrofísica de } \\
\text { Andalucía }\end{array}$ & IAA & Granada & Propio & & $89(28)$ \\
\hline $\begin{array}{l}\text { Instituto de Estudios Espaciales } \\
\text { de Cataluña }\end{array}$ & IEEC & Barcelona & Mixto & Varios & $32(4)$ \\
\hline $\begin{array}{l}\text { Instituto Mediterráneo de } \\
\text { Estudios Avanzados }\end{array}$ & IMEDEA & $\begin{array}{l}\text { Palma de } \\
\text { Mallorca } \\
\end{array}$ & Mixto & UIB & $19(9)$ \\
\hline $\begin{array}{l}\text { Laboratorio de Astronomía } \\
\text { Espacial y Física Fundamental }\end{array}$ & LAEFF & $\begin{array}{l}\text { Robledo } \\
\text { (Madrid) }\end{array}$ & Mixto & INTA & $6(3)$ \\
\hline $\begin{array}{l}\text { Laboratorio de Física de Siste- } \\
\text { mas Pequeños y Nanotecnología }\end{array}$ & LFSPN & Madrid & Propio & & $3(2)$ \\
\hline Observatorio del Ebro & $\mathrm{OE}$ & Tarragona & Propio & & $18(4)$ \\
\hline \multicolumn{6}{|c|}{$\begin{array}{l}\text { Los datos de personal incluyen a todas las personas de plantilla que constituyen el instituto. Los datos } \\
\text { entre paréntesis se refieren exclusivamente al personal científico. } \\
\dagger \text { Centro de Investigaciones Físicas Miguel Catalán } \\
\text { † Centro de Investigación en Tecnologías Físicas Leonardo Torres Quevedo. } \\
* \text { Centro Nacional de Microelectrónica. }\end{array}$} \\
\hline
\end{tabular}

Los Centros Miguel Catalán y Torres Quevedo ocupan edificios en la zona histórica del CSIC en Madrid, habiéndose reubicado entre 1995 y 1997 para aprovechar mejor los espacios disponibles. Sólo es una excepción el IAI, que dispone de instalaciones más modernas en las afueras de la capital. El OE está emplazado en el observatorio que da nombre al centro, en Cataluña. El IAA tiene un edificio que se construyó en los ochenta, y otros siete institutos (IMB, IMM, IMSE, 
IIIA, IFIC, IRII, IEEC) han inaugurado sus instalaciones a lo largo de la década de los noventa. IMEDEA está en proceso de inaugurar un edificio y sólo el IFCA ocupa unos espacios provisionales en la Universidad de Cantabria.

En general, con la excepción del IFCA, no hay otros problemas de espacio que los derivados del crecimiento de los centros. Presentan necesidades importantes el IMB y el IMSE, que tienen una alta densidad de ocupación de sus edificios respectivos, y precisan ampliar sus laboratorios y servicios para poder afrontar sus compromisos de proyectos a corto y medio plazo.

La falta de equipamiento científico no es un problema en este Area ya que, como veremos más adelante, los ingresos en función de su actividad científica y tecnológica permiten abordar su renovación sistemática. Son excepción las grandes instalaciones que comentamos más abajo, que precisan de alguna financiación complementaria de otros organismos (OCYT, por ejemplo), dado su alto coste de mantenimiento y renovación.

Además de estos institutos, que llevan a cabo investigación y desarrollo en esta Area, hay que señalar la existencia de tres instalaciones singulares que cumplen su cometido en este ámbito científico-tecnológico. La primera a considerar está conformada por la cúpula y los telescopios de que dispone el IAA en el Observatorio de Sierra Nevada. Se trata de equipos que permiten aprovechar las condiciones de elevación del suelo y limpieza del cielo que caracterizan la zona. Su ayuda a las tareas del IAA es inestimable, así como la experiencia adquirida en el instituto durante el proceso de construcción de estos telescopios.

La segunda gran instalación del área es la Sala Blanca del CNM, ubicada al lado del IMB. Se trata de la única facilidad de este tipo existente en nuestro país junto a la que mantiene Lucent Technologies en Madrid. Su objetivo es la fabricación de pequeñas series de circuitos y sensores integrados, tanto para los laboratorios de investigación como para la industria nacional. Permite hacer investigación a nivel de los diferentes pasos que integran una tecnología de fabricación CMOS, así como usar esa tecnología para probar nuevas ideas de circuito o nuevas estructuras de sensores.

Finalmente debemos citar una estructura "virtual" constituida por los servicios de gestión y apoyo al funcionamiento de REDIRIS, el eje nuclear de las comunicaciones informáticas de las universidades y centros de investigación de nuestro país. Estos servicios dependen del CSIC por encargo de la Oficina de Ciencia y Tecnología. 


\section{Física y Tecnologías Físicas}

\section{Líneas Temáticas}

Es muy difícil hacer una síntesis de los intereses investigadores e incluso de la orientación específica de cada instituto, habida cuenta del elevado número de grupos que se integran en un área tan compleja y competitiva como ésta. Sin embargo, vamos a tratar de abordar esta tarea desde la perspectiva de ayudar al lector interesado a acercarse, en una primera aproximación, a la realidad existente.

Como puede desprenderse de las observaciones hechas en el primer apartado y de los centros que se encuentran en funcionamiento, la actividad de estos institutos se puede considerar dividida entre aspectos de carácter fundamentalista y aspectos de naturaleza tecnológica.

Los primeros resumen la actividad que se dedica a alguno de los siguientes grandes tópicos de investigación:

- T1: Estructura microscópica e interacciones de la materia. Se incluyen aquí investigaciones sobre el núcleo atómico, teoría y fenomenología de partículas elementales e interacciones fundamentales, física de altas energías, materia condensada, técnicas espectroscópicas, estructura de moléculas orgánicas, interacción radiación-materia y difracción.

- T2: Organización y estructura del cosmos. Bajo este epígrafe consideramos la investigación sobre atmósferas planetarias, sistema solar, física estelar, estructura y formación de galaxias, astronomía extragaláctica, cosmología, gravitación y radiación cósmica.

- T3: Modelado de fenómenos complejos de nivel macroscópico. En este apartado pueden citarse los trabajos que se llevan a cabo relacionados con el crecimiento de interfases en medios desordenados, fenómenos caóticos y fractales en la naturaleza, física no lineal, crecimiento de cristales, codificación segura y encriptación.

- T4: Física matemática y técnicas matemáticas en Física. Además de los temas clásicos en física matemática, mecánica de medios continuos o geometría diferencial, hay que añadir investigaciones sobre inferencia, lógica difusa, lógica de la similitud, razonamiento aproximado y temporal o deducción automática, así como sobre diagramas de radiación, mejora, compresión y análisis de imágenes y secuencias o modelos del ojo humano.

Respecto a los temas de orientación tecnológica, se pueden resumir en: 
- T5: Tecnologías relacionadas con el desarrollo de equipos e instrumentos para ayudar al conocimiento de la materia. Esencialmente se trata de la participación en la concepción, diseño, construcción y explotación de diversos experimentos soportados y financiados por agencias internacionales, en especial el CERN (Centre Europeanne pour la Recherche Nucleaire). Entre estos proyectos hay que destacar las colaboraciones existentes tanto dentro del LEP como del LHC.

- T6: Tecnologías relacionadas con el desarrollo de equipos e instrumentos para apoyar la exploración espacial. Incluimos los esfuerzos de desarrollo e implementación de sistemas, tanto en estaciones de tierra como embarcados, así como la interpretación y mantenimiento de los datos proporcionados por estos instrumentos. La mayoría de los trabajos llevados a cabo están encuadrados en misiones espaciales de NASA o ESA.

- T7: Metrología. Resumimos aquí el desarrollo de nuevas técnicas de medida, el mantenimiento de patrones, los servicios relacionados con la metrología en general, así como la definición de estándares y protocolos de medición.

- T8: Tecnologías de aplicación industrial y en servicios de valor añadido. Bajo este epígrafe consideramos las tecnologías de la información y las comunicaciones, las de automatización de la producción, las tecnologías acústicas y ópticas. Cabe señalar entre ellas la robótica, la automatización de procesos, la informática, la electrónica, la microelectrónica, los láseres, la optoelectrónica, las nanoestructuras, los sensores, los ultrasonidos, así como las aplicaciones de tales tecnologías.

La Tabla II intenta dar una idea de la contribución de cada instituto a estos temas de investigación. En dicha Tabla se ha indicado con asteriscos todos los tópicos en los que un instituto tiene capacidad y actividad. Con dos asteriscos aparece destacado el tema (o temas) en el que el instituto lleva a cabo la mayor parte de su actividad.

Es interesante establecer una distribución orientativa de la capacidad que existe en el área para abordar problemas en todas y cada una de estas líneas temáticas. Para ello, en los apartados que siguen, intentaremos establecer diferentes medidas que permitan reflejar la calidad de las actividades que se llevan a cabo en el Area, estableciendo la financiación que se obtiene de los Programas de la Unión Europea, así como de las convocatorias nacionales y de contratos con empresas. De la misma forma analizaremos el número y calidad de las publicaciones. 
Física y Tecnologías Físicas

TABLA II

\begin{tabular}{|c|c|c|c|c|c|c|c|c|}
\hline & $\mathrm{T} 1$ & $\mathrm{~T} 2$ & T3 & $\mathrm{T} 4$ & $\mathrm{~T} 5$ & T6 & $\mathrm{T} 7$ & $\mathrm{~T} 8$ \\
\hline IEM & $* *$ & $*$ & $*$ & $*$ & $*$ & $*$ & & \\
\hline IMAFF & $* *$ & $*$ & & $* *$ & & & & \\
\hline IO & $*$ & & $*$ & & & & & $* *$ \\
\hline IA & & & & & & & $*$ & $* *$ \\
\hline IFA & & & $*$ & & & & $*$ & $* *$ \\
\hline IAI & & & & $*$ & & $*$ & & $* *$ \\
\hline IMB & & & & & $*$ & $*$ & & $* *$ \\
\hline IMM & $*$ & & & & & & & $* *$ \\
\hline IMSE & & & & & $*$ & $*$ & & $* *$ \\
\hline IFIC & $* *$ & & & & $*$ & & & \\
\hline IFCA & $* *$ & $* *$ & $*$ & & & $*$ & & \\
\hline IIIA & & & & $*$ & & & & $* *$ \\
\hline IRII & & & & $*$ & & $*$ & & $* *$ \\
\hline IAA & & $* *$ & & & & $* *$ & & \\
\hline IEEC & & $* *$ & & & & $*$ & & \\
\hline IMEDEA & & & $* *$ & & & & & \\
\hline LAEFF & & $* *$ & & & & $*$ & & \\
\hline LSPNT & $* *$ & & & & & & & \\
\hline $\mathrm{OE}$ & & $* *$ & & & & & & \\
\hline
\end{tabular}

\section{Potencial Científico ${ }^{2}$}

En total trabajan en el área algo más de mil personas, incluyendo el personal universitario adscrito a los Centros Mixtos. Cabe destacar el elevado número de personas que se encuentran en formación o en situación de contratado, que en 1998 era de algo menos de 500, casi el doble que el de científicos de plantilla. Afortunadamente, el éxito de las recientes políticas de crecimiento de la Institución ha significado alcanzar una situación con un mejor balance entre ambos colectivos.

En la tabla I hemos incluido los datos globales de personal existentes en cada instituto. La Tabla III nos da una idea complementaria: la 
distribución de ese personal en las diferentes funciones y escalas. En esta última Tabla hemos incluido exclusivamente el personal administrativamente adscrito a esta área, por lo que los totales son ligeramente inferiores a los que resultan de globalizar los datos expuestos en la Tabla I.

TABLA III

\begin{tabular}{|l|r||}
\hline \multicolumn{1}{|c|}{ Personal } & Número \\
\hline Profesores de Investigación & 29 \\
Investigadores & 52 \\
Científico Titular & 133 \\
Personal Universitario & 91 \\
Doctores vinculados & 19 \\
Becarios & 247 \\
Otro personal contratado & 148 \\
Titulados & 67 \\
Ayudantes y Auxiliares & 65 \\
Administrativos & 21 \\
Personal Laboral & 53 \\
Otros & 99 \\
\hline
\end{tabular}

Complementamos la Tabla III con la Figura 1, donde pretendemos visualizar las proporción de los tres colectivos en que podemos encuadrar los distintos perfiles profesionales de dicha Tabla. Es posible inferir que este personal se distribuye de forma muy razonable, con algo menos de la cuarta parte formada por el personal con capacidad de liderar investigación; casi el doble es el personal en formación, y hay un tercio del total que se dedica a tareas de ayuda técnica y administrativa.

De este personal, de su capacidad, entusiasmo y conocimientos depende el impacto que el CSIC tenga en la ciencia española e internacional en temas de física y tecnologías físicas. Por supuesto, ese impacto no es uniforme en todas las temáticas involucradas en esta área ni en todos los centros. Es difícil establecer con precisión cuál es el potencial relativo de cada una de las grandes líneas que hemos definido anteriormente. Analizando la involucración de cada instituto, el número de proyectos que cae dentro de cada tema y las publicaciones generadas, hemos determinado una distribución orientativa que, aunque debe interpretarse con cautela, da una primera aproximación cuantitativa que nos va a ser muy útil para hacernos una idea del potencial de esta área y para discutir la proyección que cabe esperar de ella 


\section{Física y Tecnologías Físicas}

en el futuro. La Figura 2 refleja la distribución del personal científico en cada una de las ocho grandes líneas temáticas que hemos presentado anteriormente.

FIGURA 1

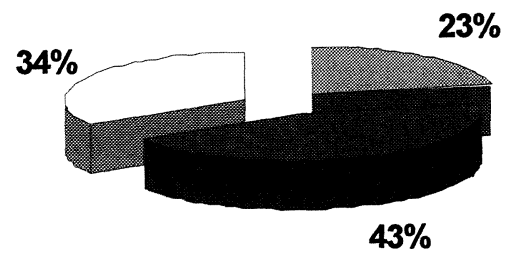

Pers. Científico

Pers. Formación

Pers. Soporte

FIGURA 2

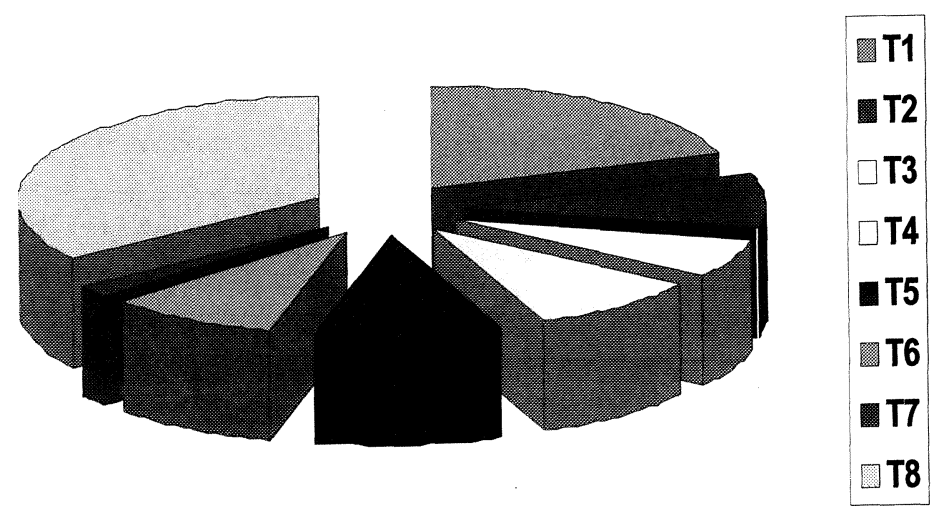

De dicha Figura es posible deducir que los dos grandes ámbitos en los que se concentra el personal investigador son los relacionados con la estructura de la materia y con las tecnologías industriales. Para interpretar mejor estos datos, en el diagrama de la Figura 3 hemos agrupado estos ocho temas en tres, motivados por nuestro interés de hacer una lectura del potencial del área en términos de los intereses de las agencias financiadoras (Programa-Marco Europeo, Plan Nacional, Planes regionales, etc). Se han agrupado los temas de carácter fundamentalista ( $\mathrm{T} 1, \mathrm{~T} 2$, T3 y T4), las tecnologías de un solo uso (T5, T6 y T7) y las tecnologías de ámbito industrial (T8). 
FIGURA 3

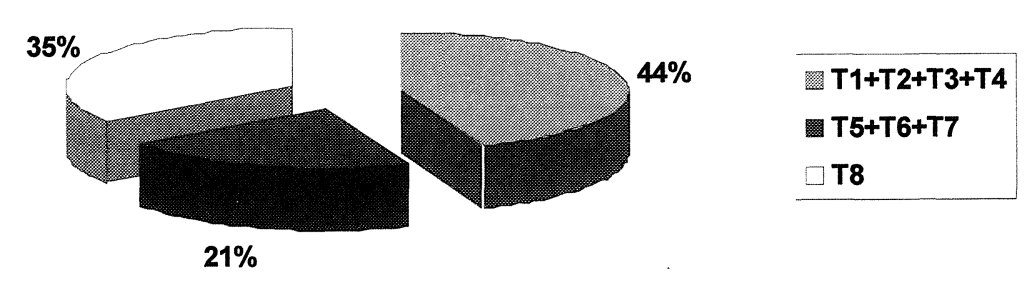

Es interesante comparar con la situación resultante de extrapolar los datos correspondientes a 1985. En efecto, si sustraemos de este diagrama los valores que dependen de los centros creados exnovo en los últimos quince años, la situación sería equiparable a la de la Figura 4. Vemos que el peso relativo de la aplicación del conocimiento a problemas tecnológicos ha crecido significativamente, lo que está en línea con el progresivo aumento de la preocupación por llevar a la sociedad los beneficios de la investigación. De nuevo estos son datos que hay

FIGURA 4
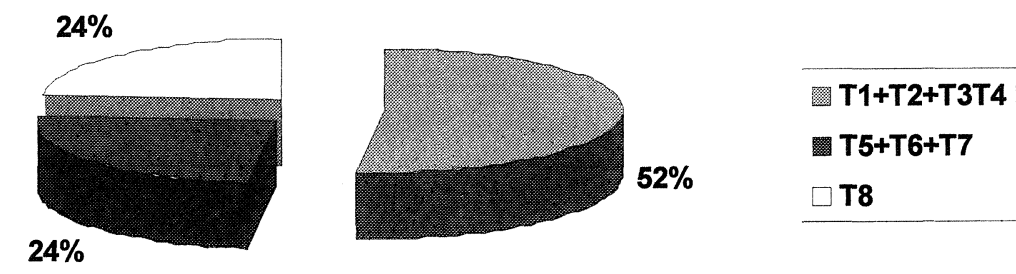

que interpretar con cuidado. De la comparación entre ambos diagramas, resulta claro que el posicionamiento tecnológico del área es ahora radicalmente mayor (56\% de los investigadores, frente a $48 \%$ en 1985), existiendo un mejor equilibrio entre la investigación realizada en temas fundamentales y la llevada a cabo en temas tecnológicos.

\section{Potencial presente $y$ futuro}

El CSIC es una institución que depende en gran medida de su propia capacidad de generar recursos económicos que puedan ser invertidos en proyectos o programas de investigación. Esta capacidad 
ha de alinearse con las necesidades manifestadas por los organismos públicos y privados que financian la investigación y el desarrollo. De ahí que sea un elemento clave en la política del CSIC su posicionamiento estratégico frente a tales necesidades.

El reto consiste en establecer un buen equilibrio entre su capacidad de hacer avanzar libremente el conocimiento y su potencial para capturar recursos económicos, en función de la respuesta que sepa dar a los requerimientos sociales y a las demandas de los sectores industriales.

Es desde esta doble perspectiva desde la que interesa analizar el potencial actual y futuro del área de Física y Tecnologías Físicas. Afortunadamente se trata de un ámbito del conocimiento en el que se incluyen líneas de investigación y desarrollo que tienen y van a continuar teniendo un gran impacto en la sociedad. En cualquier caso, vamos a distinguir tres aspectos que serán las coordenadas que han de servirnos para nuestro análisis. De una parte, vamos a considerar la producción científica del área y su incidencia en el contexto nacional. De otra, vamos a estudiar la capacidad de captación de recursos demostrada. Finalmente, tendremos en cuenta las líneas de investigación que se proponen apoyar tanto el Programa Marco de la UE como el Plan Nacional.

\subsection{Producción científica}

Dada la coexistencia dentro de este ámbito científico de temas que pueden considerarse de carácter fundamentalista, ya que apoyan el avance del conocimiento, y temas tecnológicos, que se orientan a aplicar el conocimiento, la producción (medida en base a las publicaciones generadas) debe evaluarse utilizando criterios equivalentes, en lo posible. Para hacerlo, hemos considerado las publicaciones en dos bases de datos distintas (aunque solapadas): el SCI y el INSPEC. Los temas que hemos agrupado bajo los cuatro primeros epígrafes en la Sección 2 (T1 a T4) caen completa y exclusivamente dentro de la primera de esas bases de datos. El resto, aparece en ambas o bien en INSPEC, dado que allí se registran además las publicaciones de impacto en las conferencias y congresos que son de prestigio en las áreas tecnológicas.

Siguiendo esa línea, hemos confeccionado la Tabla IV, con la que pretendemos dar una idea de la actividad y resultados de ambas orientaciones del área, medidas en parámetros equivalentes (publicaciones por doctor, en este caso). En ella hemos agrupado los institutos en 
dos bloques, lo que es una simplificación, pero nos permite de nuevo tener una visión aproximada y significativa de las contribuciones relativas.

TABLA IV

\begin{tabular}{||l|l|c|c||}
\hline & & SCI & $\begin{array}{c}\text { SCI+ } \\
\text { INSPEC }\end{array}$ \\
\hline $\mathrm{T} 1+\mathrm{T} 2+\mathrm{T} 3+\mathrm{T} 4$ & IEM, IFCA, IMAFF & 2,47 & N/A \\
\hline $\mathrm{T} 5+\mathrm{T} 6+\mathrm{T} 7+\mathrm{T} 8$ & IA, IAI, IFA, IIIA, IMB, IMM, IMS, IRII, IO & 1,51 & 2,85 \\
\hline \multicolumn{2}{|c|}{ IAA e IFIC tienen una participación significativa en ambos grupos } \\
\hline
\end{tabular}

Esta Tabla nos viene a decir que el nivel de publicación es alto, superando las dos publicaciones internacionales por doctor y año. Son valores comparables a los de otras instituciones internacionales equivalentes al CSIC.

\subsection{Capacidad de autofinanciación}

Considerando los proyectos y contratos conseguidos por las dos subáreas que hemos definido de facto en el apartado anterior podemos hacer algo análogo para establecer el potencial para captar recursos. La Tabla V refleja el número de proyectos por doctor. En esta Tabla no hemos considerado la cuantía económica de los proyectos ya que existen grandes disparidades que están parcialmente motivadas por los costes tan desparejos que conllevan estos proyectos según su temática. Con estos datos tenemos una primera medida de la competitividad y del nivel de ocupación de nuestros investigadores, que como se ve oscila entre uno y dos proyectos por doctor.

TABLA V

\begin{tabular}{|l|l|c|c||}
\hline & & $\begin{array}{c}\text { Total de } \\
\text { Proyectos }\end{array}$ & Proyectos/Doctor \\
\hline $\mathrm{T} 1+\mathrm{T} 2+\mathrm{T} 3+\mathrm{T} 4$ & IAA, IEM, IFCA, IFIC, IMAFF & 195 & 1,13 \\
\hline $\mathrm{T} 5+\mathrm{T} 6+\mathrm{T} 7+\mathrm{T} 8$ & IA, IAI, IFA, IIIA, IMB, IMM, IMS, IRII, IO & 265 & 1,86 \\
\hline
\end{tabular}

Para completar nuestro análisis, detallamos en la Figura 5 los porcentajes de financiación que corresponden a cada una de las ocho 
líneas temáticas. El total de recursos generados por año en el Area se situa en torno a los tres mil millones de pesetas. Puede apreciarse que la capacidad de generación de recursos es tanto mayor cuanto mayor es el contenido tecnológico y la proximidad a la aplicabilidad industrial.

\section{FIGURA 5}

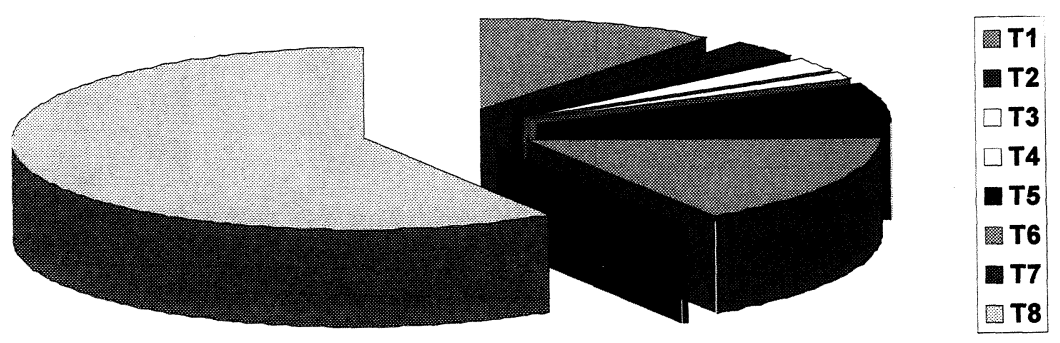

\subsection{Orientación estratégica}

La tercera coordenada que queremos considerar se refiere a la adecuación del área a las necesidades y demandas de las entidades que financian la investigación. Sin duda este es el aspecto más difícil de cuantizar. Hemos optado por determinar el número relativo de investigadores que se preocupa de temas que aparecen explícitamente en los programas de distintas agencias financiadoras. En particular hemos categorizado programas en tres grupos. Los que pertenecen al Programa-Marco, los que corresponden a las agencias internacionales (CERN, ESA, etc) y los que están relacionados con el nuevo Plan Nacional. De nuevo los resultados cuantitativos deben considerarse como un aproximación de primer orden. La Figura 6 pretende dar una visión clara de la situación. En ellos apreciamos que sólo un $40 \%$ de los investigadores de este Area trabajan en temas que son financiables por el V P-M, que menos del $20 \%$ tienen capacidad para conseguir recursos de las grandes agencias internacionales (CERN, ESA, NASA), mientras que un 60\% (prácticamente la suma de los dos colectivos anteriores) está posicionado en líneas sectoriales o prioritarias del recién aprobado Plan Nacional. 
FIGURA 6

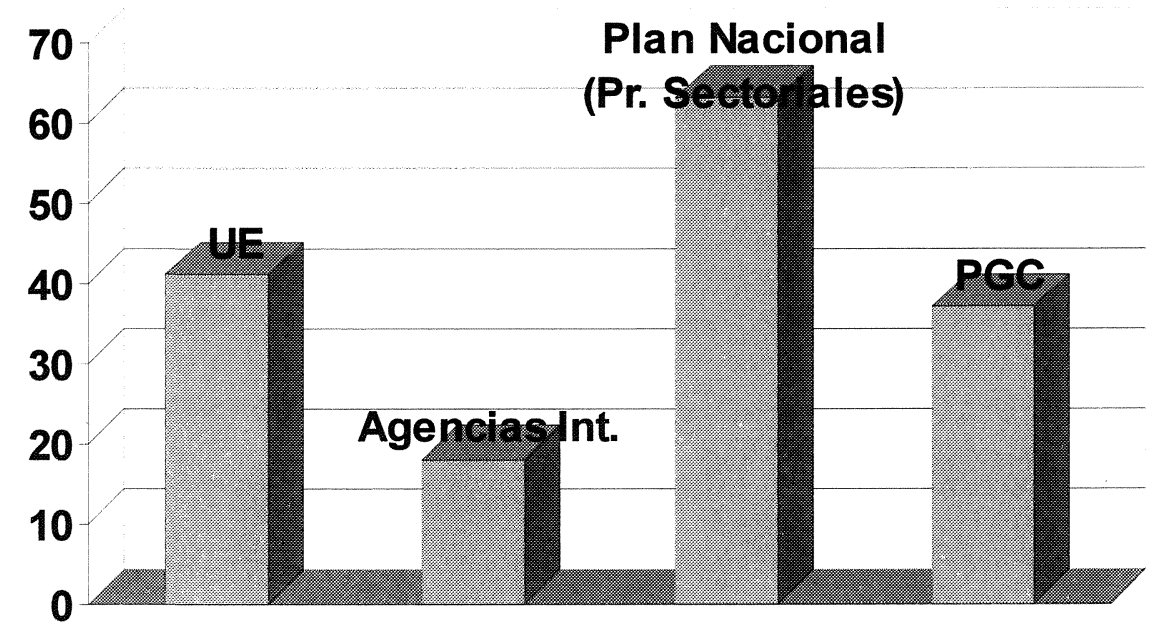

A estos datos hay que añadir que, demás de considerar en qué medida se está preparado para hacer frente a las demandas de estos planes, hay que tener en cuenta el posicionamiento estratégico del Area frente a la evolución a largo plazo de los grandes tópicos científicos, cuyo horizonte cae fuera de la visión extremadamente limitada de la planificación política. En este sentido, cabe interpretar positivamente que alrededor del $30 \%$ de nuestros científicos no estén directamente implicados en temas tecnológicos o estratégicos, que exigen una orientación a corto y medio plazo. Este tercio significa un potencial de inversión en líneas más avanzadas y novedosas, pero de mayor riesgo, lo que es una garantía para el futuro del Area.

\subsection{Discusión sobre el futuro}

En este Area hay una gran capacidad innovadora para abordar problemas científicos y tecnológicos en las líneas temáticas que hemos discutido. Pero también hay carencias significativas. Fundamentalmente podrían resumirse en la escasez de personal cualificado en informática (sólo el IIIA se dedica al completo a estos temas, aunque hay una especial cualificación en algunos otros centros tales como IRII, IMB, IMSE, IAA e IAI) o radiofrecuencia y comunicaciones en general o en servicios de valor añadido, como INTERNET, por ejemplo; temas estos últimos donde existen graves carencias en el país, que el Area 
de Física y Tecnologías Físicas del CSIC podría abordar a medio plazo si se establece un plan adecuado.

Es necesario además seguir fortaleciendo las tecnologías horizontales, ya que la situación española (comparada con la de otros paises avanzados) dista de ser la que precisa nuestro entorno económico. Sobre estas tecnologías va a continuar descansando la expansión económica y la evolución del conocimiento, de ahí su carácter estratégico. Los centros del Area relacionados con estos temas tienen un papel protagonista a nivel nacional que debe ser afianzado en el ámbito internacional.

También hay que señalar la capacidad del CSIC para abordar problemas multidisciplinares; capacidad derivada de su estructura y experiencia. Sin duda, en un futuro inmediato, los grandes desafíos científicos van a requerir conocimientos provenientes de campos del saber muy diferentes dentro del esquema tradicional, así como una componente tecnológica muy grande. Frente a esas necesidades, sólo estructuras con suficiente masa crítica, pluralidad de conocimientos, tradición de gestión de proyectos complejos y capacidad de coordinación, podrán hacer frente a esas demandas.

En cuanto a las tecnologías de un solo uso, el papel del CSIC es clave, pero convendría que se viera reforzado con una mayor implicación de otros institutos. Respecto a la Física de carácter más básico, el CSIC posee posiblemente el núcleo más numeroso del país, con una alta calidad media. Seguramente aquí la necesidad consiste en potenciar la cooperación y la complementariedad, buscando evitar un fraccionamiento caprichoso de grupos y temas que harían perder eficacia al colectivo. Líneas como las relacionadas con la formalización de la complejidad, las aplicaciones computacionales de los conceptos cuánticos o aspectos emergentes tales como la física de los seres vivos, deberían merecer mayor atención. Finalmente, aunque los problemas matemáticos involucran, de una manera u otra, a la práctica totalidad de los científicos del Area, una potenciación de las Matemáticas como tópico basal se hace necesaria y ha comenzado a ser considerada prioritaria por la Institución.

Para terminar, este Area comparte con las restantes del CSIC la necesidad de aumentar el peso del personal técnico, con un incremento sistemático y significativo de su número. Es un grave problema tanto de cantidad como de calidad. Hace falta dotar a los centros de técnicos de diferentes niveles que permitan mantener adecuadamente los equipos de laboratorio y las infraestructuras asociadas, cada vez más sofisticados y costosos, y que garanticen la calidad y fiabilidad de los datos que 
se obtengan. Pero ese grado de sofisticación y la rápida evolución de la tecnología requieren que este personal de apoyo tenga un elevado nivel de cualificación y unas necesidades permanentes de formación que deben ser atendidas. Sin ello, la comunidad científica y tecnológica de este Area va a tener dificultades crecientes, que se podrían convertir en insalvables, para cumplir con sus tareas investigadoras de forma competitiva.

\section{Notas}

1 Datos de personal de 1998.

2 Nota: datos hasta 1998 y teniendo en cuenta solamente los Centros considerados en la Memoria del CSIC como adscritos al Area. 